Original Article

\title{
Factor analysis of indicators of physical and functional preparation of basketball players
}

\author{
VALERIA TYSHCHENKO ${ }^{1}$, YAROSLAV HNATCHUK ${ }^{2}$, VIKTORIA PASICHNYK ${ }^{3}$, OLEKSIJ-OLEG \\ BUBELA $^{4}$, ZORYANA SEMERYAK ${ }^{5}$ \\ ${ }^{1}$ Department of Theory and Methods of Physical Training and Sports, Zaporizhzhia National University, \\ UKRAINE \\ ${ }^{2}$ Department of Physical Education, Khmelnytskyi National University, UKRAINE \\ ${ }^{3}$ Department of Sports and Recreational Games, Lviv State University of Physical Culture, UKRAINE \\ ${ }_{5}^{4}$ Department of gymnastics, Lviv State University of Physical Culture, UKRAINE \\ ${ }^{5}$ Department of Fencing, boxing and national single combat, Lviv State University of Physical Culture, \\ UKRAINE
}

Published online: Octomber 31, 2018

(Accepted for publication September 05, 2018)

DOI:10.7752/jpes.2018.s4269

\begin{abstract}
The problem of physiological study of non-traditional means of training attracts a lot of scientists' attention in recent years. This is due to the need to provide a maximum development of certain muscle groups and direction of the training process. Proper use of simulators weight machines bring positive results. Obviously, an important step in the use of technology is the right choice for team sports coaches and personal trainers for the effectiveness of the training process. One effective component of this process is the inclusion to a training program with the help of physical exercises would be TRX® function loops. Among specialists of functional and physical training innovation as TRX $\AA$ - training is one of the best equipment for complex solution of the tasks. This exercise is an exercise using body weight. It was designed to develop strength, power, core stability, flexibility and balance. TRX® is the valuable creation for the sports industry of any levels. TRX® Training Suspension Training became commonly usable for Fitness Health Clubs, Professional Sports and Elite Strength Conditioning Workout for Sports Teams. Therefore, it was suggested the use of TRX® exercises in preparation of basketball players. The results of the study clearly demonstrated that the inclusion in the training program for basketball players training with functional loops TRX ${ }^{\circledR}$ contributed to a significant increase in the level of their overall physical performance and aerobic capacity of the organism. Utilization of TRX $®$ in the training process can be considered as an important factor in increasing the efficiency of preparation in basketball.

Key words: basketball, TRX® functional loops, training process, objective, method
\end{abstract}

\section{Introduction}

Suspension Training (TRX $\left.{ }^{\circledR}\right)$ is considering as the best option for both beginners and professional athletes because it's easy to operate and manage. For example, Nicholas Ratamess describes the body weight exercises that are highly popular and the suspension training or total body weight resistance exercise (TRX®), that is used in school- and community-based programs to track progress, develop personalized programs, provide motivation, and assess the effectiveness of a fitness program (Ratamess, 2012). In addition, working with elderly participants, many of whom with multiple chronic injuries, and required supervision to create modification that could help them maintain and improve performance without aggravating any preexisting conditions, Suspension Training was introduced into their regular training routines (Dawes, 2017; Gaedtke \& Morat, 2016). Further, the research was conducted, using TRX®, among 36 health but untrained men. Scientists were focused on exercises that could improve strength, power, speed and jumping ability. However, after the study was completed, the scientists determined there was no significant improvement (Maté-Muñoz et al, 2014). In effect, very few scientists have compared the effects of balance on performance measures in training programs performed under both unstable and stable conditions (Sparkes \& Behm, 2010). Thus, exploring results of wrestlers 10-12 years old suggest that TRX ${ }^{\circledR}$ functional loops can be used in the training program, as this tool increases: strength, speed-strength performance muscles in the arms and legs. However, muscle growth, as indicated, is insignificant that is valuable for wrestlers who limited weight category (Miroshnikov et al, 2011). During the research on the effectiveness of training for qualified handball players, changes were identified and aerobic physical performance of athletes for two leading microcycles with TRX ${ }^{\circledR}$ assistance to conduct four major exercises for the lower body (Humstring Runner, Side Lunge, Overhead Squats). This has led to positive dynamic (Tyshchenko, 2014). However, in the study of a particular sport, such as basketball, scientists were focused on finding out if three weeks of $\mathrm{TRX}{ }^{\circledR}$ suspension training were enough to enhance static balance in junior 
basketball players. Those three weeks of TRX ${ }^{\circledR}$ training seemed to be insufficient for modifications to occur in the static balance of junior players (Iuliana et al, 2015).

It is necessary to generate explosive muscular forces very rapidly to accelerate, change directions, and then reaccelerate over relatively short distances in Team Spots (Duthie et al, 2006; Valeria \& Olexander, 2015; Evhen \& Valeria, 2017). Therefore, maximal velocity is likely a greater predictor of success, and the ability to change velocity over short distances in multiple directions based on game- specific stimuli is prevailing (Dawes $\&$ Lentz, 2012). Because of that, athletes must apply greater muscular forces into the ground to overcome the oppositional forces of gravity to produce velocity (Kovacs, 2012). In addition, the analysis of scientific methodic literature defied that there is a deficit of sport science and movement-specific functional resistance training data on strength and conditioning for basketball players at every level. Therefore, it requires a deep analysis of every action would be taken and influenced on basketball.

Recently, experts in various sports widely used factor analysis technique that reveals the complex dominant components (Mihnov, 2015; Morozova, 2017; Tishchenko, 2016). This causes the athletic performance, determines the nature of the relationship between the indicators. Also it allows determine the contribution of individual factors to the overall result. Relevant is the definition of the factor structure of preparedness in basketball, which is an extremely important task. However, the literature wasn't paying enough attention to this question. Establishing main components for preparedness would greatly enhance the effectiveness of their training and competitive activity.

\section{Materials \& methods}

Participants. The study involved 20 basketball players between the ages of 19 to 30 years old Super League team of Ukraine «Zaporizhzhya-ZOG».

Goals and procedures. The goal of this study was to use TRX ${ }^{\circledR}$ to determine the effect on the functional readiness for basketball players. To solve this goal, we have the following tasks:

1. Evaluate the level of physical and aerobic performance in a training process for basketball players.

2. Analyze the dynamics of physical condition and aerobic capacity of basketball players' primary and experimental groups.

3. Arrange the leading factors of functional readiness for basketball players.

Overall design. The training in both groups was within the base stabilizing mesocycle speciallypreparatory phase. The control group of 10 athletes was involved in accordance to the traditional training program. Conventional exercises that meet the stated objectives of each part were used. The experimental group of 10 engaged involving specially designed complex exercises using TRX ${ }^{\circledR}$ equipment. Each exercise was performed 45 seconds, break - 15 seconds, every round - by 2 sets. The break between sets -1 minute. The total duration of one set -7 minutes. The total duration of the training -45 minutes.

The study was approved by the local research ethics committee, performed in accordance with the ethical standards of the journal, IJSM and conformed to the recommendations of the Declarations of Helsinki. The current study was undertaken in Ukraine after approval by the Institutional Research Ethics Committee at Zaporizhzhia National University. Participation was voluntary; participants received no incentives. They were provided with information about the aims, objectives and methods of the study before the start of monitoring. Data was anonymous and confidential, and data protection was observed at all the time. Each basketball player gave voluntary informed consent for the inclusion in the study.

Measuring methods. Estimated overall physical performance using the method Astrand - determined level of overall physical performance, aerobic power and capacity. In the beginning and at the end of the preparatory and competitive macrocycle periods of basketball players, both groups were assessed by level of physical capacity and aerobic performance using submaximal test $\mathrm{PWC}_{170}$. Exact and relative values were calculated $\mathrm{PWC}_{170}\left(\mathrm{aPWC}_{170}, \mathrm{kgm} / \mathrm{min} ; \mathrm{rPWC}_{170}, \mathrm{kgm} / \mathrm{min} / \mathrm{kg}\right)$ and maximal oxygen consumption (aMOC, $1 / \mathrm{min} ; \mathrm{rMOC}, 1 / \mathrm{min} / \mathrm{kg}$ ), coefficient of efficiency of blood supply (CEBS), the power index (PI), index hypoxia (IH).

Statistical analysis. Generalization of the studied characteristics was assessed by mean arithmetic value, standard deviation, error of mean arithmetic and factor analysis. Confidence of differences between mean values was stated by Student's t-criterion. Assessment of statistical hypothesizes based on 5\% significance level. For statistical processing of data, we used licensed program Microsoft Excel (2010). Statistical analysis of the received results was conducted, considering recommendations on Microsoft Excel tables' usage for computer data analysis. The study applied a method to find the graph of ordinal function based on the use of the adjacency matrix (Demukrona algorithm) (Ore, 1965). The topological sorting of the graph allows to determine the importance of each element and its impact on the functional readiness for competitive activity in general.

The prime component analysis was used to identify the most significant factors functional preparation for basketball players. The advantage of this method was the possibility to limit the most informative main components and exclude others in the analysis, which has greatly simplified the interpretation of the data.

\section{Results of the research}


Preliminary physical fitness testing of basketball players who participated in the study made it possible to establish the following. As seen from the results in early training in preliminary and experimental groups were characterized by substantially equal values and overall physical performance aerobic productivity (Table 1). Levels aPWC ${ }_{170}, \mathrm{rPWC}_{170}, \mathrm{aMOC}$, and $\mathrm{rMOC}$ matched low value. In general, the pre-test results determined the insufficient level of physical fitness of the surveyed groups (Table 1).

The conclusive evidence of the above results was the data relative changes in the study of physical performance and aerobic productivity for basketball players in the control and experimental groups. In the analysis of the functional state of the body on the subject to maintain the overall physical performance, it can be stated that for the period of rest not the adaptive stereotypes embodied for the past seasons have been lost. Therefore, with an average energy at the beginning of the season, the leading role of aerobic mechanisms in central and vice versa could be saved, to ensure the efficiency of the anaerobic organism in point guards laid down as a result of the training process, as well as according to their physiological characteristics. As a matter of fact, the implementation of the training program leads to a significant increase in performance and efficiency of power supply arrangements for the players. It should be noted for preservation of specific stereotype for all players' positions in basketball.

Further, to summarize the last phase of the survey, the attention should be paid about the rising level of fatigue and the partial depletion of energy systems. It should be emphasized that this decline is not dramatic and disastrous nature of all the indicators of power supply is not reduced to a level that allows speak of exhaustion and inability to maintain physical condition of basketball players.

The results obtained in all phases made it possible to determine the orientation of quality changes in the studied parameters during the basketball season, the quantitative characteristics and the possibility of correction in training and competitive process for greater optimization and an increase in the functional state of the organism. This tendency is to increase the total of all indicators of physical performance and energy mechanisms including the competitive level, maintaining the level of these indicators in the first half of the season and reduce them to the background processes, developing due to fatigue at the end of the season.

Table 1. Dynamics of indicators of functional preparation for basketball players during the annual macrocycle

\begin{tabular}{|c|c|c|c|c|c|c|c|c|c|}
\hline Indicators & BPP & EPP & $\begin{array}{c}\mathrm{t}_{\text {BPP- }} \\
\text { EPP }\end{array}$ & BCP & ECP & $\begin{array}{c}\mathrm{t}_{\text {BCP- }} \\
\text { ECP }\end{array}$ & $\begin{array}{c}\mathrm{t} \\
\text { BPP- } \\
\text { BCP }\end{array}$ & $\begin{array}{c}\mathrm{t} \\
\text { EPP- } \\
\text { BCP }\end{array}$ & $\begin{array}{c}\mathrm{E} \\
\text { EPP- } \\
\text { ECP }\end{array}$ \\
\hline \multicolumn{7}{|c|}{ Control group } \\
\hline $\mathrm{aPWC}_{170}$ & $1564,6 \pm 23,6$ & $1622,4 \pm 27,2$ & 1,60 & $1624,7 \pm 26,5$ & $1562,8 \pm 26,4$ & 1,66 & 1,69 & 0,06 & 1,57 \\
\hline $\mathrm{rPWC}_{170}$ & $17,18 \pm 0,15$ & $17,42 \pm 0,18$ & 1,02 & $17,19 \pm 0,14$ & $17,12 \pm 0,11$ & 0,39 & 0,05 & 1,01 & 1,42 \\
\hline $\mathrm{aMOC}$ & $4550,1 \pm 59,3$ & $4685,1 \pm 52,5$ & 1,70 & $4710,04 \pm 62,2$ & $4534,65 \pm 67,2$ & 1,92 & 1,86 & 0,31 & 1,76 \\
\hline $\mathrm{rMOC}$ & $49,4 \pm 0,30$ & $51,4 \pm 0,6$ & 2,98 & $50,54 \pm 0,44$ & $48,92 \pm 1,22$ & 1,25 & 2,14 & 1,16 & 1,82 \\
\hline \multicolumn{7}{|c|}{ Experimental group } \\
\hline $\mathrm{aPWC}_{170}$ & $1578,2 \pm 27,8$ & $1726,86 \pm 31,9$ & 3,51 & $1668,2 \pm 29,3$ & $1558,84 \pm 24,3$ & 2,87 & 2,22 & 1,35 & 4,19 \\
\hline $\mathrm{rPWC}_{170}$ & $17,11 \pm 0,18$ & $18,74 \pm 0,18$ & 6,40 & $18,17 \pm 0,17$ & $17,08 \pm 0,15$ & 4,81 & 4,28 & 2,30 & 7,08 \\
\hline $\mathrm{aMOC}$ & $4542,05 \pm 61,3$ & $4869,1 \pm 70,2$ & 3,51 & $4740,04 \pm 64,6$ & $4499,44 \pm 53,5$ & 2,87 & 2,22 & 1,35 & 4,19 \\
\hline $\mathrm{rMOC}^{2}$ & $49,5 \pm 0,38$ & $53,12 \pm 0,37$ & 6,83 & $51,88 \pm 0,35$ & $49,53 \pm 1,26$ & 1,80 & 4,61 & 2,43 & 2,73 \\
\hline
\end{tabular}

Notes: BPP - the beginning of the preparatory period; EPP - the end of the preparation period;

$\mathrm{BCP}$ - the beginning of the competition period; ECP - the end of the competition period

Therefore, participants of the experimental group during the study were characterized by a significantly higher magnitude $\mathrm{aPWC}_{170}$ gain values, $\mathrm{rPWC}_{170}$, aMOC and $\mathrm{rMOC}$, basketball players than the control group (Table 1). According to the algorithm method of prime component, the accuracy of survey results is achieved if the percentage of the sample is substantially between interconnected elements is not less than $60 \%$ of the total variance. When analyzing the factorial study of functional readiness for basketball players, the structure value of mentioned values in all groups exceeds the desired range, which was $78 \%$. One of the key points of our study was to determine the relationship between the level of functional manifestations of athletes and the effectiveness of physical fitness using TRX®.

The table 2 evidenced how interconnected TRX ${ }^{\circledR}$ certain exercises between each other, and the level of manifestation for functional readiness. Factor analysis procedure allowed to generate the system load factor and determine the three factors of physical fitness, eigenvalues were higher than one (Table 2). In general the composition factor (whose contribution to the total dispersion made $16.3 \%$ ) entered parameters which characterize TRX® exercise for the upper body: Roll up $r=0,870$; Table Row $r=0,912$; TRX ${ }^{\circledR}$ Twist $r=0,886$. The second factor $(20.4 \%)$ clustered index, which reflected the TRX® exercises for the thighs and buttocks: 
TRX ${ }^{\circledR}$ Frog $r=0,920$; Sprinter Start $r=0,784$; Wide Jump $r=0,755$. The third factor $(41.3 \%)$ was above the threshold load variables that reflect TRX ${ }^{\circledR}$ exercise for the upper and lower body: Burpee $r=0,926$; Push up + Tuck Knee $r=0,868$; Mountain Climber $r=0,880$.

Table 2. The grouping and distribution of the elements of physical fitness for basketball players by using the primary method component

\begin{tabular}{|l|l|c|c|c|}
\hline \multirow{2}{*}{ № } & \multirow{2}{*}{ Components } & \multicolumn{2}{|c|}{ Factors } \\
\cline { 3 - 5 } & & F1 & F2 & F3 \\
\hline x1 & aPWC 170 & 0,323 & $\mathbf{0 , 6 5 6}$ & $\mathbf{0 , 7 8 2}$ \\
\hline x2 & aMOC & 0,449 & $\mathbf{0 , 7 2 1}$ & $\mathbf{0 , 5 6 8}$ \\
\hline x3 & CEBS & $\mathbf{0 , 7 5 7}$ & 0,217 & $\mathbf{0 , 8 4 1}$ \\
\hline x4 & PI & 0,245 & $\mathbf{0 , 5 9 5}$ & $\mathbf{0 , 6 9 8}$ \\
\hline x5 & IH & $\mathbf{0 , 6 4 2}$ & 0,331 & $\mathbf{0 , 5 8 7}$ \\
\hline & \% & 18,3 & 20,4 & 61,3 \\
\hline
\end{tabular}

Note: The factor loadings are significant $(\mathrm{p}=0.05)$ when the absolute value is not less than 0.532

Subsequent graphic analysis of the influence factors on the selected parameters $\mathrm{Fi} \ldots \mathrm{Xi}$ possible to determine it in the form of a bipartite graph (chromatic number is 2), since a plurality of vertices can be broken into two parts $\{\mathrm{F}\}$ and $\{\mathrm{X}\}$, so that neither one vertex in $\{\mathrm{F}\}$ is not connected with vertices $\{\mathrm{F}\}$ and no vertex in $\{\mathrm{X}\}$ with vertices $\{\mathrm{X}\}$ (the subset of vertices $\{\mathrm{F}\}$ and $\{\mathrm{X}\}$ called lobes bipartite graph $\mathrm{G}$ ). This clearly shows the effect of each factor on the performance aPWC 170 , aMOC, CEBS, PI, IH (Figure 1).

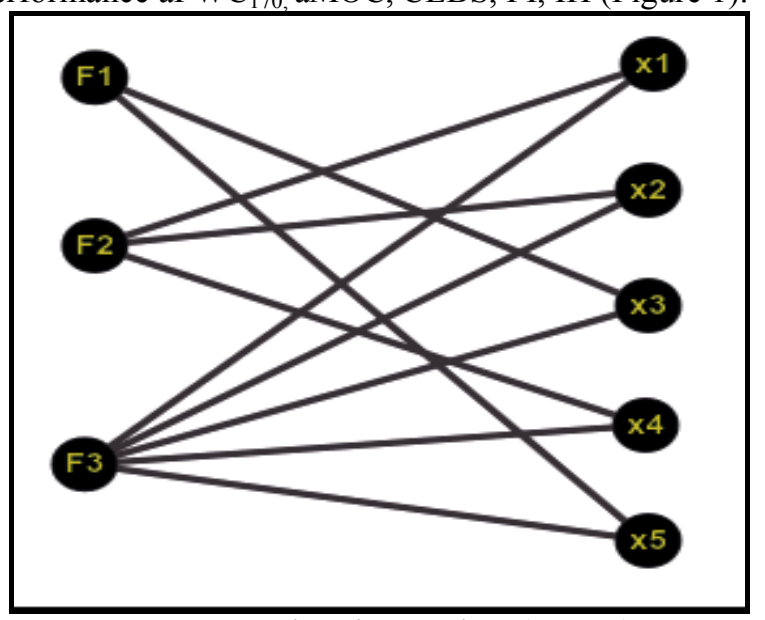

Figure 1. Bipartite graph $\mathrm{G}\left(\mathrm{V}_{\mathrm{FX}}, \mathrm{E}\right)$

Values of $\mathrm{x} 1, \mathrm{x} 2$ and $\mathrm{x} 4$ are determined by the combined effect of F2 and F3 factors and indicators $\mathrm{x} 3$ and $\mathrm{x} 5$ - the combined effect of factors F1 and F3. Optimization graph based on the weight of vertices allowed us to determine that the graph contains a Eulerian circuit: F3 - x1 - F2 - x2 - F3 - x5 - F1 - x3 - F3 - x4 - F2 (Figure 2).

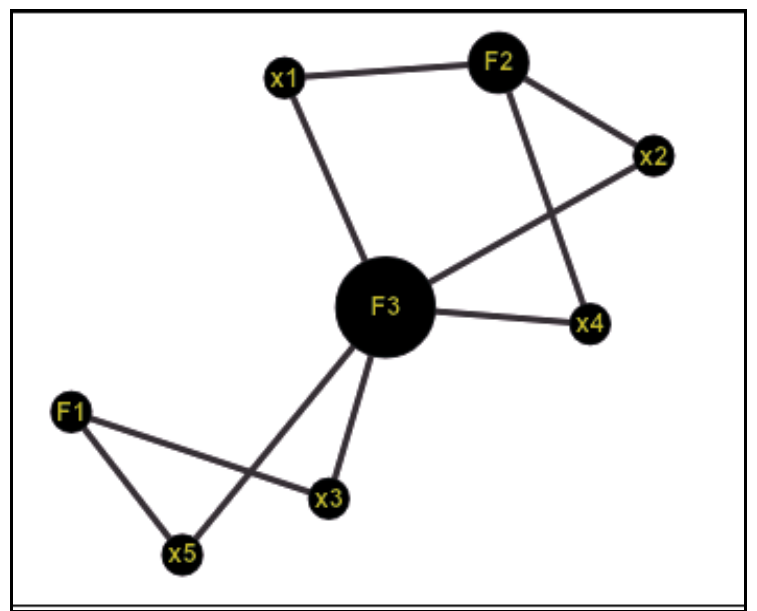

Figure 2. Graphical representation of the optimized graph $\mathrm{G}\left(\mathrm{V}_{\mathrm{FX}}, \mathrm{E}\right)$ based on the weights of the vertices

That confirms that the graph is connected (Euler cycle if and only if the graph is connected) and, accordingly - the impact of the factors identified in studied parameters. Further optimization of the graph $G$ $\left(\mathrm{V}_{\mathrm{FX}}, \mathrm{E}\right)$ allowed to determine the maximum degree of the graph vertices $\mathrm{k}_{\mathrm{F} 3}=4$, confirming the influence of the factor $\mathrm{F}_{3}(\mathrm{TRX} \mathbb{R}$ exercise for the upper and lower bodies) (Figure 2). 


\section{Discussion}

A number of experts in the field of theory and methodology of sports training indicate that the modern technology of preparation of top class athletes is based on the results of scientific research, and has scientific and methodological platform (Ratamess, 2012; Yuriy et al, 2016; Valeria et al, 2017). Various scientists have developed biomechanical and psycho-pedagogical bases of an activity for athletes, as well as key aspects of their training: technical, physical, psychological and theoretical (Kovacs, 2012; Evhen \&Valeria, 2017). Thus, numerous studies suggest that a scientific approach to the training qualified athletes capable of providing high achievements (Valeria \& Olexander, 2015).

Basketball is a dynamic and explosive sport, full of intensity and contacts. That's the reason why a lot of basketball players can perform elements of the game, such as dunking the ball or quick movements through defenders and dribble to the net effortlessly. All those aspects take the huge influence on the athlete's body. With the use of Suspension Training exercises the player can simulate the way his body moves on the court, making him more able to handle challenges of practices and matches. By the variety of training and uneven load of work on the body, the TRX ${ }^{\circledR}$ Trainer better develops core strength and stability element to the required moves which can help the basketball players to be stronger and have better balance on the court.

In recent years, due to the intensification of the struggle on the international arena of Ukrainian basketball Teams, the problem of training competitive basketball players has acquired a special significance. Further, over the past several Olympic cycles established tendency was focused on systematic increase of the complexity of technical and tactical actions. To solve the problem and to achieve better results, the questions were aroused to use innovative technologies. In this regard, of particular importance and urgency of the problem was to train and prepare highly qualified basketball players. In particular, the Ukrainian basketball is urgent requirements to fulfill and implement the elements of higher complexity and their impact on the competitive outcome. They are either not exist or cannot be included in the preparation of the structure depending on the coaching staff.

The coach's main purpose of the practice is to concentrate on the players physical abilities developing their speed, strength and explosive power to make sure they have a good balance and able to jump high, etc. The biggest importance to joint stability is not necessarily strength or endurance but motor control. Instability resistance exercises promote co-contractions, increasing joint stability (Behm et al, 2011). The idea is to involve the whole athlete's body during the practice, and how capable it is to challenge the ball and work under the net on the basketball court. TRX ${ }^{\circledR}$ allows the coach to create training programs for the players using progressing and regressing movements for strength, conditioning, stretching, rehabilitation, and to give additional bust to their bodies needed on scheduled practices or before competitions.

Suspension bodyweight training has many advantages. Moreover, many benefits it has brought to sports performance particularly in basketball. Jungle Gym, TRX®, Rotational Bodyweight Trainer from CrossCore180 ${ }^{\circledR}$ helped bring this form of training to the forefront of the sports performance and fitness industries. The latter of which has an added component of rotation with a greater equalization. These are all great options that may increase an athlete's strength through a full range of motion, while utilizing a fully portable unit for convenience (Hess\&Camacho, 2014). Using TRX ${ }^{\circledR}$ Training programs would benefit basketball players in order to achieve better balance and coordination on the both sides of the hardwood. The programs could vary from Beginner to Advance depending on the athletes' physical condition. TRX ${ }^{\circledR}$ assists the athletes to develop and perform basketball movements, concentrate on specific muscles and improve training outcomes. Based on these facts, several exercises TRX® are proposed that could be valuable as a part of the training program designed to increase preparedness of basketball players.

\section{Conclusions}

The results of the study clearly demonstrated that the inclusion in the training program for basketball players training with functional loops $T R X ®$ contributed to a significant increase in the level of their overall physical performance and aerobic capacity of the body. In our study factor analysis allowed us to determine the presence of intra-relationships between indicators of functional and physical preparedness Super League basketball team of Ukraine «Zaporizhzhya-ZOG».

Obviously, the use of TRX ${ }^{\circledR}$ can be considered as a factor in increasing the effectiveness of the training process. The main advantage - is to work with its own weight, without the additional complication that allows the athlete to remove the vertical load. In the study, analyzed dynamics allows for the correct use, to make changes at all stages of the training process as a whole team and as individual players, who have higher tendency.

\section{Acknowledgments}

The authors are grateful to the coaches and basketball players «Zaporizhzhya-ZOG», who participated in the study. Valeria Tyshchenko was supported scholarship during $\mathrm{PhD}$.

Conflicts of interest - The authors declares that there is no conflict of interests. 


\section{References}

Behm, D. G., Drinkwater, E. J., Willardson, J. M., \& Cowley, P. M. (2011). The role of instability rehabilitative resistance training for the core musculature. Strength \& Conditioning Journal, 33(3), 72-81.

Dawes, J., \& Lentz, D. (2012). Methods of developing power to improve acceleration for the non-track athlete. Strength \& Conditioning Journal, 34(6), 44-51.

Dawes J. (2017) Complete Guide to TRX Suspension Training. Human Kinetics. 216 p.

Evhen, P., \& Valeria, T. (2017). Peculiar properties and dynamics of physiological indicators in Handball team. Journal of Physical Education and Sport, 17(1), 335.

Duthie GM, Pyne DB, Marsh DJ, and Hooper SL. (2006). Sprint patterns in rugby union players during competition. J Strength Cond Res 20: 208-214,.

Gaedtke, A., \& Morat, T. (2016). Effects of Two 12-week Strengthening Programmes on Functional Mobility, Strength and Balance of Older Adults: Comparison between TRX Suspension Training versus an Elastic Band Resistance Training. Central European Journal of Sport Sciences and Medicine, 13(1), 49-64.

Hess, S., \& Camacho, C. (2014). Rotational basketball movements. Strength Training, 1(3), 22.

Iuliana, B.B., Grațiela-Flavia, D. E. A. K., Simona, M., \& Adrian, P. (2015). TRX suspension training method and static balance in junior basketball players. Educatio artis gymnasticae, 27-34.

Kovacs M. Training for Linear Acceleration. Colorado Springs, CO: NSCA Hot Topic Series. Available at: http://www.nscalift. org/HotTopic/download/Speed\% 20Training.pdf. Accessed: May 7, 2012.

Maté-Muñoz, J. L., Monroy, A. J. A., Jiménez, P. J., \& Garnacho-Castaño, M. V. (2014). Effects of instability versus traditional resistance training on strength, power and velocity in untrained men. Journal of sports science \& medicine, 13(3), 460.

Mihnov A. Analysis of the structure of technical and tactical and psychological preparation for highly qualified hockey players by principal component, Young sport science of Ukraine. 2015. V.1. P. 153-160.

Miroshnikov, A., Nesterov, P., Pashkin, P., Tabakov S. \& Myakinchenko, E. (2011). Using the training device TRX functional loops in the physical preparation of sportsmen-wrestlers 10-12 years. Lika, 117-121.

Morozova E.A. (2017) Factor analysis of the components of athletic performance for the strongest speed skaters in the world, $500 \mathrm{~m}$ distance. Pedagogical-psychological and medical-biological problems in Physical Education and Sports, Volume 12, № 1, 22-28.

Ore O. Graphs and their application. Moscow: Mir; 1965. 174 p.

Ratamess, N. A. (2012). ACSM's foundations of strength training and conditioning. Wolters Kluwer Health/Lippincott Williams \& Wilkins. 229-232.

Sparkes, R., \& Behm, D. G. (2010). Training adaptations associated with an 8-week instability resistance training program with recreationally active individuals. Journal of Strength \& Conditioning Research, 24(7), 1931-1941.

Tishchenko, V.A. (2016). Skilled handball player functionality variation in annual macrocycle. Theory and Practice of Physical Culture, 3, 72-73.

Tyshchenko, V.A. (2014). The use of innovative technologies in education-training process of handballers. Visnyk of Chernigiv National Pedagogical University. S.: Pedagogical Sciences. Physical education and sports. 118;4; 211-4.

Valeria, T., \& Olexander, P. (2015). Control of general and special physical preparedness by qualified handballers. Journal of Physical Education and Sport, 15(2), 287.

Valeria, T., Pavel, P., Olena, B., Lia, G., Maria, S., Anna, S., \& Olga, S. (2017). Testing of control systems of highly qualified handball teams during the annual training macrocycle. Journal of Physical Education and Sport, 17(3), 1977-1984.

Yuriy, B., Maryan, P., \& Valeria, T. (2016). Dynamics of changes in the functional state of qualified handballers during macrocycle. Journal of Physical Education and Sport, 16(1), 46. 